\title{
Models for Character Animation
}

\author{
Gordon Collins and Adrian Hilton \\ Centre for Vision, Speech and Signal Processing \\ University of Surrey, Guildford GU25XH, UK \\ g.collins,a.hilton@surrey.ac.uk \\ http://www.ee.surrey.ac.uk/Research/VSSP/3DVision
}

\begin{abstract}
We present a review of methods for the construction and deformation of character models. We consider both state of the art research and common practice. In particular we review applications, data capture methods, manual model construction, polygonal, parametric and implicit surface representations, basic geometric deformations, free form deformations, subdivision surfaces, displacement map schemes and physical deformation.
\end{abstract}

\section{Introduction}

The science of computer character animation addresses the problem of building models from some captured data and some physical knowledge of the character. Three stages are involved; static model design, deformation scheme and motion prescription. The static model may be entirely captured (from a range scan, for example) or entirely user specified (using a commercial model building package, for example). Deformation of the model requires at least some degree of physical knowledge. If an animator wants the deformations of his character to be smooth then in effect he is approximating the physical process of skin stretching by a smooth surface. On the other extreme animators may want a more physically realistic model which computes the deformations of every muscle and skin movement. Motion laws too may be painstakingly applied by an animator moving a stick man skeleton or may come directly from motion capture data. The problem for the animator then is, given some captured data, how to best use his knowledge of physical movement?

The methods for character animation are various and dependent on the application and the amount and kind of captured data. The trade off involved are 
essentially realism, speed and compactness. Computer games require models that can be rendered very quickly and may need some form of collision detection; a notoriously CPU hungry practice. As a result realism must often take a back seat. At the other extreme, film animators require a high degree of believability and perhaps realism but their models do not need to be rendered in real time. Applications for animation are discussed in Section 2.

The application usually determines the type of data capture needed and hence the representation used. In Section 3 we discuss the model building approach which requires no data capture and the scanning approach where models are copies of real objects. In Section 4 we outline the representations; polygons, parametric surfaces and implicit surfaces.

An important concept that unifies most animation schemes is the layered approach. This is a very natural approach since bodies which are layered in the sense that they have a skeleton, a muscle layer, a fat layer and a skin layer. Each layer is controlled only by the previous one. While some animation systems exactly mirror this anatomical organisation (see Section 5.7) it is more normal to have a hierarchy that goes something like; skeleton, low resolution layer, high resolution layer. As with the anatomically accurate model, moving a skeleton, in turn moves the higher layers. Unlike the human body, it is the skeleton that moves the muscles since it is easy for animators to move a point on a skeleton. The purpose of a layered approach is to go from a complicated model of a character to a simple skeleton which can be easily animated. As an example, the layered animation scheme in $[80,81]$ is shown in Figure 1.

\section{Layered Animation}

Model Construction High-resolution Model Capture \& Construction

- 3D Data Capture (hand-held sensor)

- Geometric Fusion \& Optimisation

\section{Skeleton Layer}

- Skeleton Fitting

- Motion Control Design

Low-resolution Control Model Layer

- Point-to-line Mapping

- Low-resolution Control Model Animation

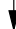

High-resolution Model Layer

- Point-to-surface Mapping

- High-resolution Model Animation

Figure 1: System overview 
The problem then lies in how to translate skeleton movement into rendered surface movement. Here, again, a variable degree of physical realism is used. In Section 5.7 we discuss methods which are concerned with modeling muscles and skin with springs and solving the resulting physics based differential equations. Such a practice is clearly time consuming. Where physical realism is neither required nor practical, some other, believable, law of deformation is needed. This law is almost always that deformations must be smooth. Two ways of achieving this, free form deformations and subdivision surfaces are also discussed in Section 5.

In this paper we present a review of literature on the model building and deformation of characters, we do not concentrate on the actual animation of a model. This may come from a variety of sources, whether they be motion capture techniques (common in the computer games industry) or if they are in some degree autonomous (AVATAR technology).

\section{Applications}

The current applications for character animation are roughly, web-based animation, computer games and films. Each application has different requirements which are;

- Web-based animation - Compression and CPU efficiency.

- Computer games - CPU efficiency.

- Films - Realism.

Web-based animations must be transmitted quickly and so compression is important here. It is, also, desirable to transmit the animation in a coarse form first and then successively finer forms. For this, web-based animation makes use of level of detail (LOD) algorithms and progressive transmission [47].

Computer game characters need to be animated quickly and rendered in realtime. Often the details of a character are less important than their motion and their interaction with other characters and objects. Because of this, computer game characters are nearly always constructed while the captured data comes in the form of motion capture.

In contrast, film characters are required to be believable and perhaps also realistic. Characters do not have to be rendered in real time and so may be very complicated. The task for the researcher is to automate some of the painstaking work that an animator has to put in. A common technique is to build characters from scans of clay sculptured characters. Motion is usually dictated by an animator rather than by applying captured motion. Deformations must be believable and 
this is done, as we will see later, either by applying physical laws or by ensuring smooth deformations.

\section{Data Capture}

Character models require different degrees of 3 or 4D data capture and hence different levels of manual input. This ranges from a full scan of a character together with motion capture for animation rules, to a manually constructed model which is animated by manually moving control vertices.

\subsection{Model Construction}

3D model construction has become a grown up industry with several glossy magazines (digit, 3D world) and web sites [1,3] concerned with the art and technology of constructing models. There are numerous CAD packages for model building with 3D Studio MAX, Maya3D and Lightwave being the standards. However working on a $2 \mathrm{D}$ computer screen to create $3 \mathrm{D}$ characters is not an easily mastered task and this is often discouraging for artists. Despite the maturity of 3D software tools the process of manual shape construction can be highly time consuming to achieve visual realism for detailed characters such as those used in film animation.

Interactivity is the key here and Turner et al. have addressed this problem [85] by augmenting the desktop with a head tracking sensor and special glasses. Another, well studied, solution is haptics [51]. A haptic device is a handle with several degrees of freedom. The handle can receive force feedback when a virtual intersection occurs so that the user can easily navigate with it around a virtual enviroment which is constrained by the objects in it. Haptic devices provide a more natural interface to sculpt the surface shape of a 3D computer model or character.
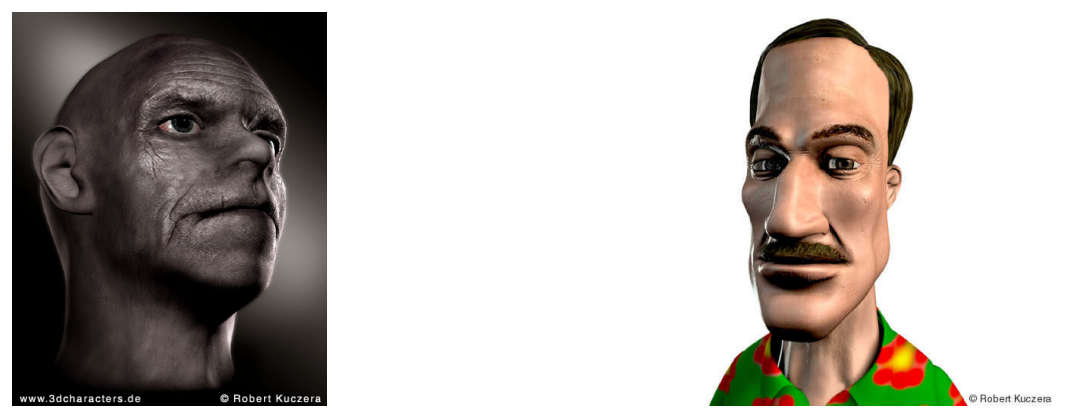

Figure 2: Characters created by Robert Kuczera in Maya and 3d Studio Max 

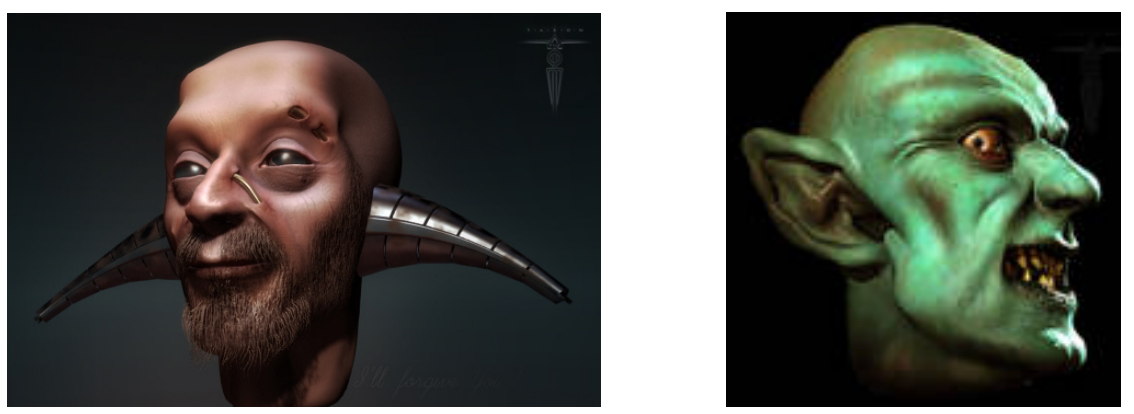

Figure 3: Characters created by Taron in Lightwave.

\subsection{Range Data}

An easier and more natural way for an artist to work in 3D is to sculpt a character out of clay. The surface shape can then be captured using either a touch probe or optical range sensor to measure the 3D location of points on the surface. Range sensors have the advantage of requiring no surface contact and allowing rapid measurement of thousands of points on the surface. The sensor projects a structured light pattern onto the object surface and captures a camera image of the projected pattern. Optical triangulation between the projector and camera is used to reconstruct the distance of points on the surface from the camera as a 2.5D "range image". A number of established technologies exist for range measurement including a single laser stripe which is swept across the surface, grey code image sequences of binary stripe patterns and random binary dot patterns. In recent years highly accurate scanners have become commercially available and are the norm for range data capture.

Passive techniques for shape capture reconstruct the surface shape from a set of multiple view images. Shape-from-silhouettes [70, 30] has become an established technique for recovering the approximate surface shape of an object by imaging against a known background, such as a blue-screen. Images from multiple views are combined to determine the spatial volume occupied by the object and reconstruct a surface model. This approach can be used to produced highly realistic object models by texture mapping. However, in isololation the silhouette based approach can not reconstruct surface concavities. Close-range photogrammetric approaches have been developed which reconstruct 3D shape from matches between images [4]. Matches of image features between images are obtained either manually or automatically to recover models of surface shape. Extensive research in computer vision community has addressed the issue of automatic matching be- 
tween images for structure recovery [29, 39]. Photogrammetric approaches result in accurate strcture recovery but sparse feature data due to the requirement for visually distinct features on the object surface. Reconstruction of surface models from such data remains an open-problem. Dence matching techniques have also been used to recover dence 3D surface measurements [29, 50]. However, due the problems of automatic matching these are relatively inaccurate compared to active sensors. Recently model-based approaches for recovering 3D shape from passive multiple view images have been introduced where domain specific knowledge is used to constrain the reconstrcution. Model-based approaches have been developed to recover visually realistic models of architecture [20], faces[32] and people [40]. This use of model-based approaches together with photogrammetric techniques for recovering structure from video image sequences offers a promising technology for future highly realistic model building.

For example, a hand-held range sensor system, 3DScanners' ModelMaker [2], was used by Hilton et al. to build animation models [41, 42, 81]. The sensor is a laser stripe based range sensor with a six degree-of-freedom position sensor. The user may move freely around an object to capture the data and use his knowledge of the object to position the sensor effectively (an advantage over automatic sensors). The result is a dense triangular mesh which is structured in such a way that no two points are connected if they are not within a certain threshold distance of each other. Thus the topology is recreated, see Figure 4. A similar system was recently used by FrameStore to capture the models for a recent BBC series 'Walking with Dinosaurs' (http://www.bbc.co.uk/dinosaurs).

Whichever capture technique is used the problems involved are integrating different overlapping views (for example different sweeps of the laser scanner) into one surface, dealing with missing data and dealing with outlying data points. This problem is subdivided into a problem where the connectivity of measured points is known (the structured problem) and where it is not known (the unstructured problem). The structured problem has been well studied [18, 43, 76, 84, 79]. The single surface may be reconstructed by fusing layers that coincide in a specified volume constructed from the surface $[18,44]$. The general problem of surface reconstruction of unknown objects from unstructured 3D point remains an openproblem [11, 25, 46, 65, 66]. A recent paper [73] deals effectively with outlying or missing data by a hybrid method which augments scanned data with a hierarchy of user-specified models. 


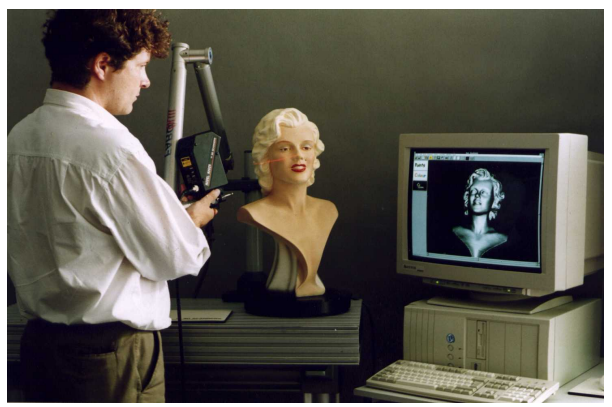

(a)ModelMaker system

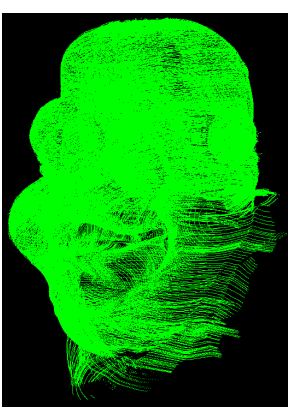

(b) Raw data

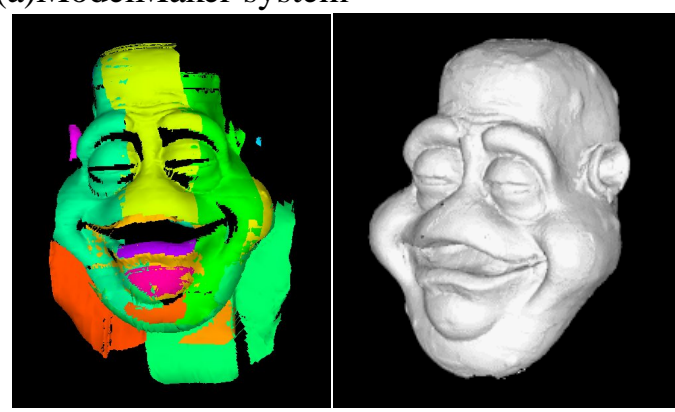

(c)Mesh patches(partial) d) Fused model

Figure 4: Model reconstruction from hand-held sensor data

\section{Representation}

The method of model building usually dictates the representation of a model. Constructed models are usually represented by parametric surfaces whereas captured data usually comes in the form of a point cloud which lends itself to polygonalisation. The pros and cons of both representations and a third representation, metaballs, are discussed here and are well documented in Watt and Watt [87].

\subsection{Polygons}

A polygonised surface is usually a collection of vertices which are connected into triangles or quadrilaterals. Besl [10] gives a good overview of the advantages of polygonal representations for various applications and analyses. The most notable advantages are due to the fact that polygons are a very simple building block. Because of this a wide range of topologies can be approximated by polygons. Also rendering polygons is very simple and efficient as it is supported directly by current graphics hardware. The disadvantage is that many vertices may be needed 
to approximate a smooth surface. Also polygonal surfaces cannot be deformed in an arbitrary way. For example, if a triangular surface is twisted too much then eventually two edges will cross so that the connectivity is violated.

\subsection{Parametric Surfaces}

Parametric surfaces can be roughly characterised as curves with a predetermined smoothness, which fit some given control points automatically. They are simple to use as the modeler may specify just a few control points in order to specify a large surface. Parametric surfaces are widely used by animators since they can be smoothly deformed simply by moving a single control point. Parametric surface models are usually built by fitting together different patches of parametric curves. The difficulties occur in the manual task of trimming and fitting together these patches so as to maintain smoothness over the join. A further difficulty is that parametric surfaces cannot be directly rendered but must first be discretised (into a polygonisation for example) to be rendered.

There are many parametric surfaces [28]; Bezier surfaces, B-Splines, $\beta$-Splines, NURBS which all differ in terms of ease of rendering, how locally they are affected by control points and how smooth they are. B-Splines are popular as control points have a very local influence and they are easily patched together. NURBS, NonUniform Rational B-Splines, are a generalisation of B-Spline and have become the computer graphics standard.

\subsection{Subdivision Surfaces}

The traditional distinction between splines and polygons has been muddied in recent years with the arrival of robust subdivision surface techniques which are a polygonisation but also a smooth surface. Subdivision surfaces appear to be the best of both worlds and have been used extensively in a modern paradigm of character animation, "Geri's Game" [21] by Pixar animation studios. The use of subdivision in animation is described in more detail in Section 5.5.

\subsection{Implicit Surfaces}

An entirely different approach is used by Thalmann et al. [78, 5] and Desbrun and Gascuel [22]. They construct implicit surfaces which are mathematical field functions, $f(\mathbf{r})=$ constant, giving the distance of a surface from a point $\mathbf{r}$. A typical choice of the field function is $1 /(r-c)^{2}$. Thalmann et al. attach an ellipsoidal volumetric primitives, "metaballs", to the joints of a skeleton and then blend them 
together using B-splines (see Figure 5) As the skeleton moves, the metaballs deform accordingly, making this an excellent approach to muscle modeling. Very few primitives are needed to construct a model, however rendering and model construction is far from straightforward.

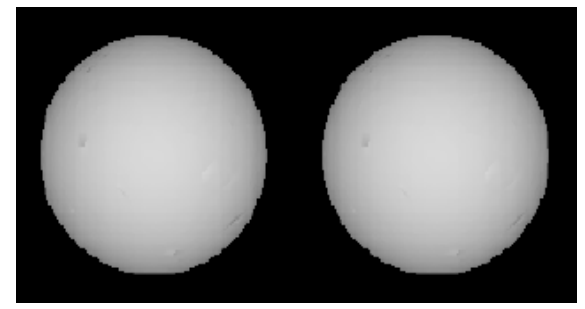

Figure 5: a) Spherical implicit surfaces

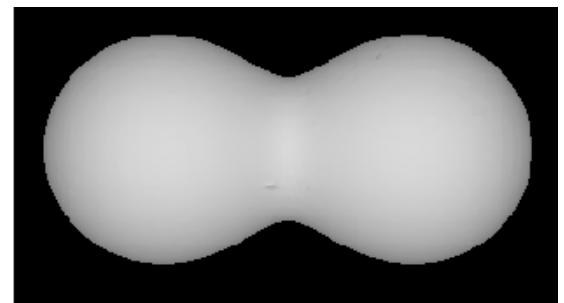

b) Surface blended together

\section{Deformation}

Once a static model is built, specifying how regions deform requires some extra input. For most animators the manipulations are done in a straightforward geometric way, such as directly manipulating the model, interpolating between known poses or using a simple skeleton model. More sophisticated models use smooth deformation methods such as free form deformations (FFDs) or subdivision surfaces. Physically realistic models are usually too computationally expensive for today's computers but are a very active area of research. These methods are all discussed here in order of complexity.

\subsection{Direct Methods}

All the representations mentioned above can, to a certain extent, be directly animated by moving control points or vertices. In the case of parametric curves a single control point can be moved to locally effect the shape of a whole region. For polygonisations direct animation of individual vertices is a painstaking but common practice. Furthermore, polygonal vertices may only be moved with regard to their connectivity or else edge crossing may occur.

One step up from direct methods are the global deformations of Barr [8]. He generalises the standard matrix transformations (translation, rotation etc.) so that the matrices may change depending on where they are applied. In this way a wider range of shapes and deformations is possible than with the standard deformations. Chang and Rockwood [15] generalise further by allowing the axes on which the 
transformations act to change, for example the transformations may act on an axis which is a B-spline. Although a wide range of deformations are possible in this way, these methods cannot perform all the deformations that animators may require.

\subsection{Basic Skeleton Methods}

The problem of moving many polygonal vertices or parametric control points may be solved by imposing a simpler layer within the model and animating that. Maestri [62] gives a review of the technical details of this process as well as some of the packages available for it. A basic form of this layered approach is widely used and involves fitting a simple "stick-man" type skeleton may be placed inside the model. Figure 6 shows a generic avatar model animation sequence which is deformed using a skeleton.

The standard $\mathrm{H}$-anim format [75] for animating virtual humans has a joint hierarchy which is reviewed in [6]. Similarily MPEG-4 is a standard format for faces which uses a similar parametric deformation method [71].

The problem is one of how to attach surface vertices or control points to a skeletal surface. A poor attachment may lead to surface collapses, bulging and the wrong segment being mapped after the skeleton is animated. For example, if a skeletal segment influences all its nearest vertices, then, when it is animated, parts of one leg may move as the other leg. Researchers $[63,81]$ have looked into this problem and produced automatic attachment which counter all these problems for simple one-chain joints. Complicated joints such as the pelvis and especially the shoulder are open problems which are currently tackled with physically modelled constraints [64].

\subsection{Shape Interpolation}

Shape interpolation is a common method for animating faces, which is closely related to the technique of morphing. Essentially a library of expressions is built and an animator may move between expressions in the library by interpolating. The method is clearly limited by the size of the library and also by the exact method of interpolation. Maestri [62] gives the technical details of this method, some practical tips and presents the packages that perform it. Lewis et al. [59] present a state of the art method of shape interpolation and also unify this with skeletal deformations. They show that where skeletal deformations break down, the face and shoulder for example, shape deformation may take over and give a realistic deformation. 

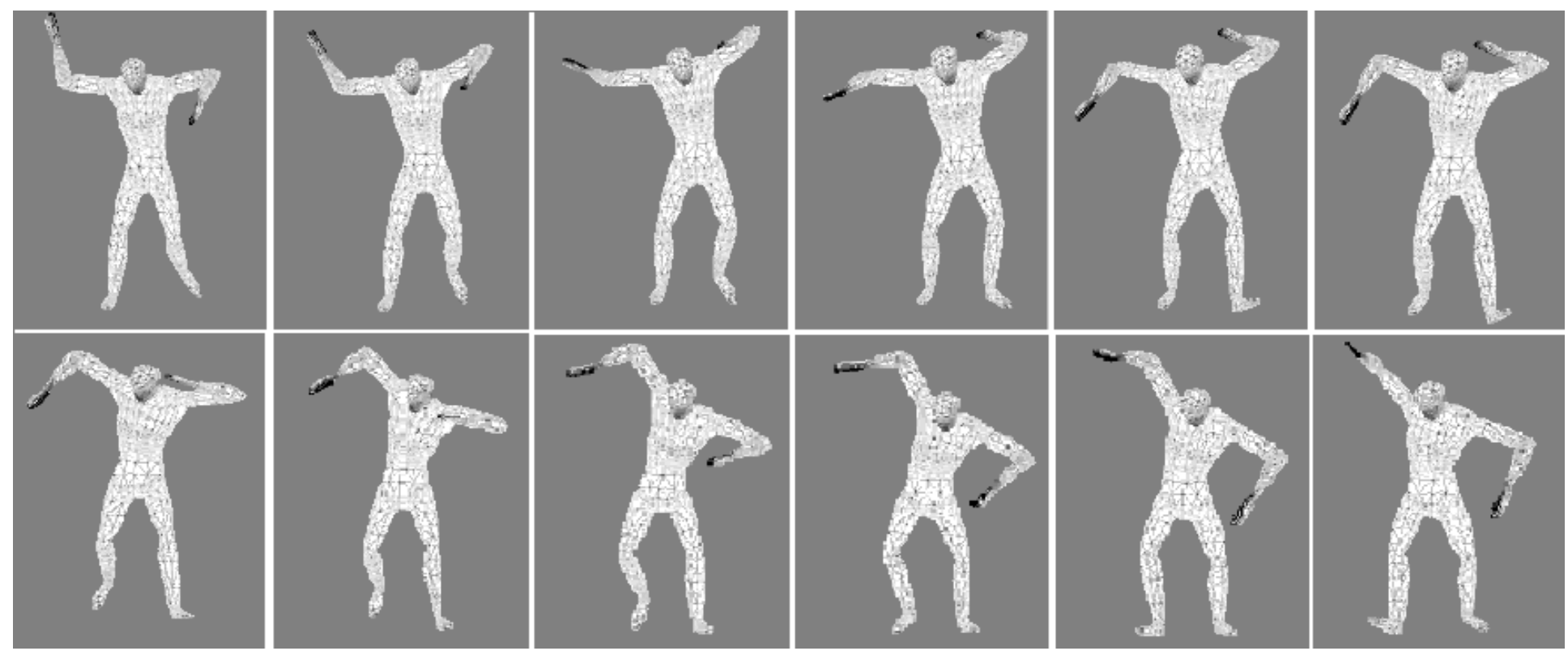

Figure 6: Skeleton based animation sequence for an avatar model.

\subsection{Free Form Deformations}

Free Form Deformation (FFD) is a class of methods which are independent of representation. They were first developed by Sederburg and Parry [77] for deforming soft objects. Sederburg uses the analogy of embedding the object in deformable plastic. When the plastic is deformed, the object, which is also to be considered as flexible, is deformed accordingly. An object is enclosed within a four dimensional parametric curve, for example a Bézier hyperpatch, which is a volume (usually a cube). The control points of the hyperpatch are deformed and, since the hyperpatch is a smooth function, the object is deformed in a smooth way (see Figure 7) If the hyperpatch were simply a volume, instead of a parametric curve, then the deformation would be equivalent to simply moving control points on the object.

Recent developments in the method have concentrated on fitting FFDs to objects $[9,38,68,72]$. The Extended Free Form Deformation method of Coquillart $[16,17]$ welds together standard FFD blocks to create a toolkit of new primitives which can be better fitted to an arbitrary topology. MacCracken and Joy [61] subdivide an FFD lattice into a sequence of lattices which converge to the shape of the object. 

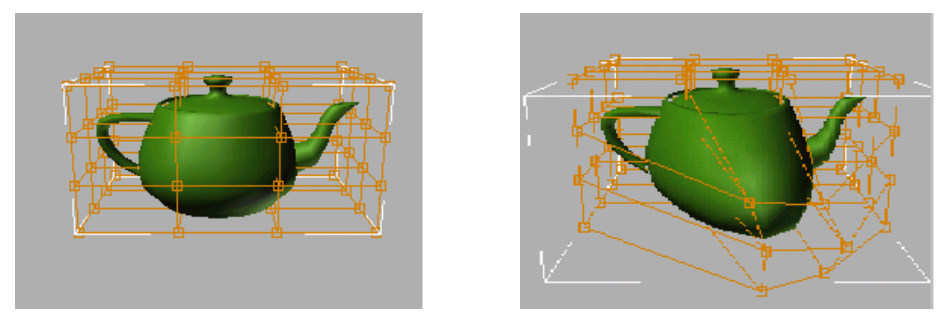

Figure 7: Free form deformation applied to a teapot

Comprehensive animation systems with heavy use of FFDs are described by Faloutsos et al. [27]. Chadwick [14] presents a system in which a skeleton moves the control points of FFDs to deform muscles and physical spring laws move control points on fatty areas such as cheeks. Other work is concerned with maintaining physical properties through FFDs such as volume preserving methods [45, 74].

\subsection{Subdivision}

A growth area in computer graphics of recent years has been subdivision surfaces. Subdivision surfaces are a way of obtaining a smooth surface with a polygonal representation. Starting with a coarse polygonal mesh, new vertices are added and, in some schemes, old vertices are moved. The resulting refined mesh is a smooth surface in the sense that, if this process is repeated ad infinitum, the "limit surface" would be a spline surface. Figure 10 shows a head model after 2,3 and 6 subdivisions of the whole mesh.

The methods to perform subdivision are categorised into interpolating and approximating schemes. Approximating schemes insert new vertices and move existing ones. The new points are generally inserted in a geometrically simple manner (in the centre of a triangle or splitting the side of a triangle). Smoothness is achieved by "relaxing" the old points by repositioning them at an average of the surrounding points. Interpolating schemes have the desirable property that their original control points are not moved. In order to ensure smoothness, the template of control points needed to compute the position of the new vertex must be larger.

Charles Loop [60] described the archetypal approximating scheme for triangular meshes. In his scheme each edge of a triangle is split in half and the resulting three points are connected to form four triangles. The old vertices are then relaxed. Dyn et al. [24] proposed the interpolating Butterfly scheme which was extended to arbitrary meshes by Zorin et al. [90]. Other seminal schemes are Catmull-Clark [13] and Doo-Sabin [23] for quadrilateral meshes. Also worthy of note is the $\sqrt{3}$ - 
subdivision scheme of Kobbelt [52] in which a vertex is placed in the middle of a triangle and then connected to each vertex, creating three triangles. The edges of the original triangle are then swapped to connect each new vertex, in this way undesirable thin triangles are avoided. The advantage of this novel scheme is that, unlike many schemes, refinement may be localised and therefore it is a useful tool for adaptivity.

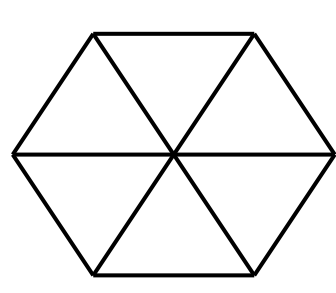

a) Original Mesh

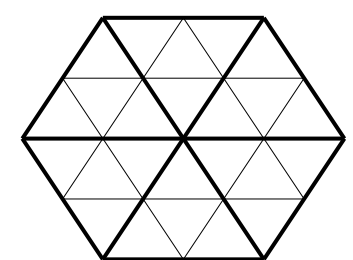

b) New Vertices Inserted

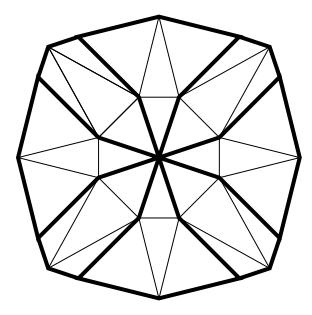

c) Vertices relaxed

Figure 8: Representation of Loop Subdivision

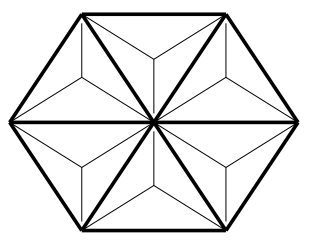

a) New Vertices Inserted

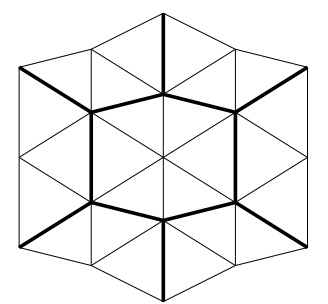

b) Edges Swapped

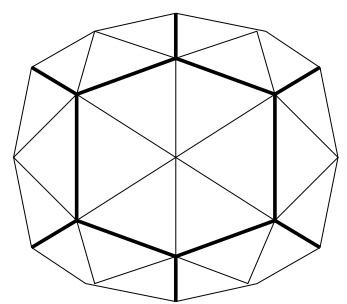

c) Vertices relaxed

Figure 9: Representation of $\sqrt{3}$-Subdivision

DeRose et al. [21] make extensive use of Catmull-Clark type subdivision surfaces for a sophisticated commercial animated cartoon. They favour subdivision surfaces over parametric surfaces because of the "considerable manual effort" involved in fitting these to each other and in keeping them fitted without visible seams appearing. They also use a variant of subdivision surfaces due to Hoppe et al. [48] which allows sharp edges to be present in the surface. 

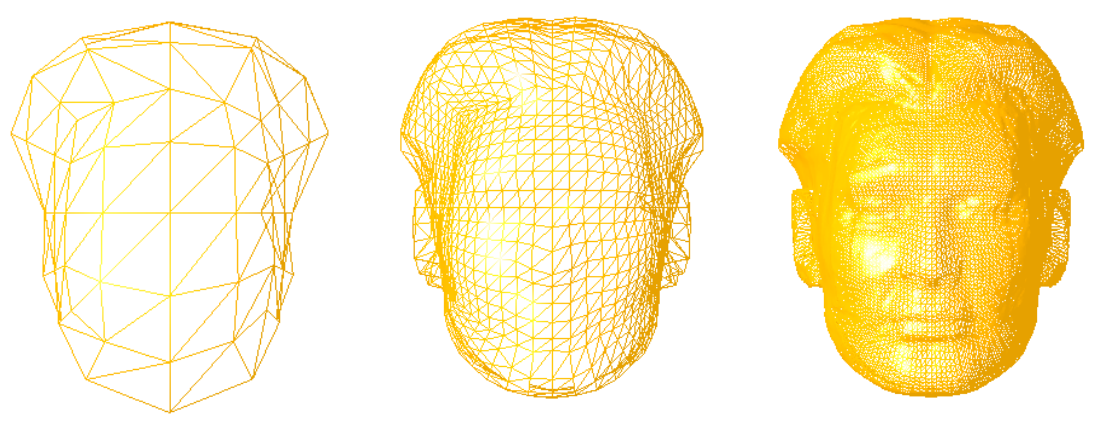

(b) Reconstructed head models at three $\operatorname{LOD}(N=2,3,6)$

Figure 10: Reconstructed subdivision head model at three levels-of-detail

Subdivision surfaces provide an easily implemented representation which is compatible with polygonal meshes. They share all the advantages of parametric representations (smoothness, few control points) but are easily rendered and do not have to be patched together. Adaptive methods are needed to counter the problem of overly dense meshes.

\subsection{Displacement Maps}

In order to display detail on a model (such as detailed scanned data) an extra layer in the hierarchy is needed. This may be represented by a displacement map. A displacement map is generated from a detailed point $\mathbf{p}_{\mathbf{d}}$ by computing a normal $\mathbf{n}$, a distance $d$ and a point of intersection on the control layer $\mathbf{p}_{\mathbf{c}}$ such that

$$
\mathbf{p}_{\mathbf{d}}=\mathbf{p}_{\mathbf{c}}+d \mathbf{n}
$$

The detailed layer can then be reconstructed by subdividing the control mesh and rendering the model at the corresponding displaced point. The normals used must be continuous on the control model to ensure all detail points can be mapped and that the detailed layer varies smoothly as the low resolution mesh is deformed.

Krishnamurthy and Levoy [56] ensured a continuous normal by calculating displacements off a B-Spline surface. Hilton et al. [80, 81] reconstruct a captured detailed surface from a coarse control model by computing a displacement map for each detailed point along a normal to the coarse mesh (see Figure 11). Once the mesh is deformed the detailed layer can be reconstructed by subdividing and remapping. The map is calculated in such a way as to preclude detailed layer intersections and seams. A similar approach is followed by Lee et al. [57]. The 
difference here is that the displacement map is calculated on a subdivision surface generated from the control mesh.

Displacement maps allow multiple level of detail representations simply by specifying how many times the control model should be subdivided. This is a very useful tool for web based animation since it allows progressive transmission of levels of detail and effective compression of the model.

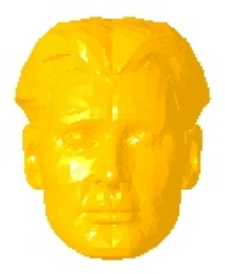

a) High-resolution Model

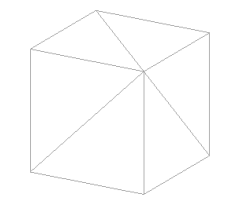

b) Control mesh

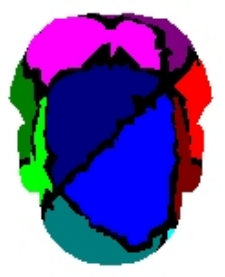

c) Colour coded mapping

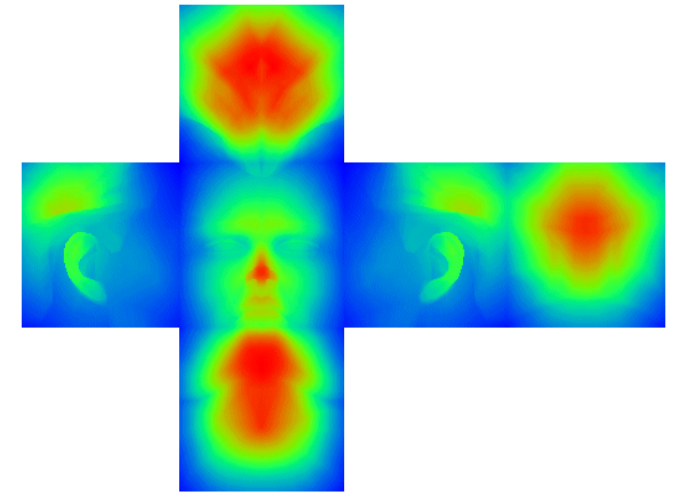

d) Displacement map with colour representation of height

Figure 11: Head model mapped onto a cube

\subsection{Physically Based Animation}

Physical animation methods are as diverse as their applications. Researchers have tackled problems in everything from finger nails and clothes to breasts and buttocks. In fact, modeling muscle and skin layers is now a mature subject. Usually some spring model is set up connecting muscles, skin and skeleton. Newton's laws of motion and Hook's law of springs can be used to derive differential equations for the skin and muscle motion. A simple model for a particle, $\mathbf{r}$ with mass $M$, damping coefficient $\gamma$, gravitational constant vector $\mathbf{g}$ and coefficient of elasticity 
$k$ might be stated as,

$$
M \frac{d^{2}}{d t^{2}} \mathbf{r}(t)=k(\mathbf{r}(t)-\mathbf{r}(0))-M \mathbf{g}-\gamma \frac{d}{d t} \mathbf{r}(t) .
$$

The terms on the right hand side are due to elastic, gravitational and damping forces respectively. Often elastic properties between particles are considered and then a connectivity matrix of forces is added to the model. A further sophistication is the nonlinear effect of allowing the coefficient of elasticity $k$ to vary with its extention. This leads to a system of ordinary differential equations which are solved numerically. The problem is constrained by collisions between skin and skin and between skin and object.

An example of a fully intergated physical system is that of Grobbetti and Turner [85]. They present an interactive system called LEMAN. The model has skeleton, bone and muscle, fat and skin layers. The skeleton layer is a stick Figure which can be moved and in turn deforms a bone and muscle layer. Fat is modeled simply as a layer of constant thickness with connective tissue in between the skin and muscle. The skin is then deformed by considering forces from the muscles via connective tissue as well as elastic, damping and gravitational forces. This requires solving a system of differential equations using a finite difference numerical technique. Figure 12 depicts a similar framework.

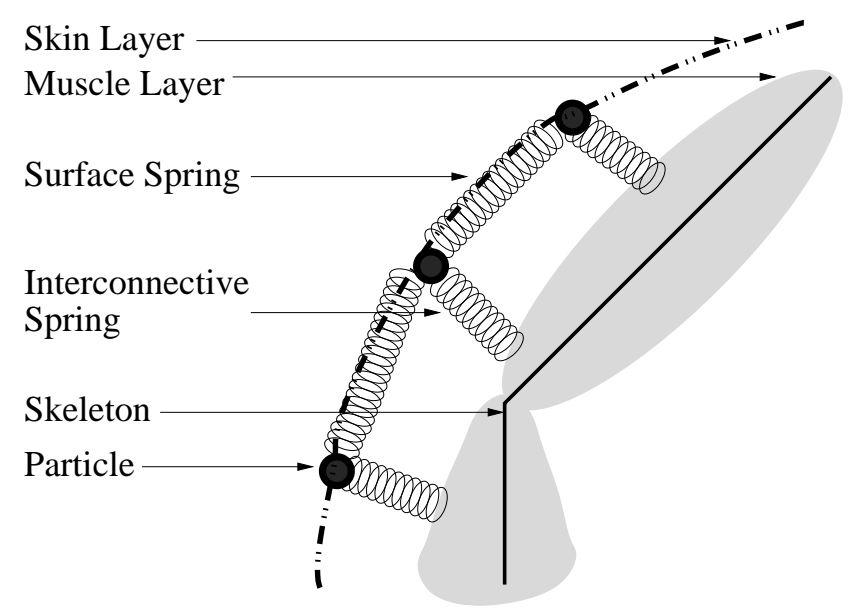

Figure 12: Layered model with springs

Among the earlier physically realistic layered models were Komatsu [55] who adjusted parametric surface control points according to joint angles on a skeleton. Forsey [31] follows this approach but adds a hierarchy of control for detailed 
movements. Chadwick et al. [14] are also pioneers in this field (see Section 5.4) as is Gascuel [33] who used a simplified and computationally faster method. She attaches one end of a spring to a muscle and the other to the control points of a B-Spline skin layer. This also allows springs to affect nearby springs. Terzopoulos [82] invented dynamic NURBS whereby differential equations for Lagrangian dynamics are solved with a finite element method to move the control points of a standard NURBS.

Thalmann et al. [5, 69] use metaballs in a layered physical approach to muscle deformations They use a realistic skeleton and use designers to model each individual muscle. Wilhelms and Gelder also propose a working anatomical model which with a minimun of computation [88]. Physical modeling has been strongly focused on facial animation by Lee et al. [58], Magnenat-Thalmann et al. [26] and Koch et al. [53, 54]

An alternative to the mass spring model, is to consider the skin as an elastic surface whose shape is dictated by minimising its elastic energy. Koch et al $[53,54]$ write the total energy as,

$$
E=\int_{\Omega} \alpha \text { Stretching }+\beta \text { Bending } d \Omega .
$$

if we consider a surface $w(u, v)$ and no external forces, then

$$
\begin{gathered}
\alpha \text { Stretching }=\alpha_{1} w_{u}^{2}+\alpha_{2} w_{v}^{2}+\alpha_{3} w_{u} w_{v} \text { and } \\
\beta \text { Bending }=\beta_{1} w_{u u}^{2}+\beta_{2} w_{v v}^{2}+\beta_{3} w_{u v}^{2} .
\end{gathered}
$$

The energy is minimised over the surface $d \Omega$ which gives the shape of the surface. The minimisation is usually done using the Finite Element Method (FEM), whereby piecewise polynomial functions are defined on a triangulated surface such that the functions fit together at their boundaries and this constructed surface minimises the energy function above. James and Pai [49] present the subject well and use a novel solver for the minimisation. Gourret et al. use this model for grasping [34].

In most systems, physically realistic detail is patched on to an existing model. Examples of this kind of modeling are numerous. Magnenat-Thalmann et al. have researched clothes [83, 36, 86], hair [19, 37] and wrinkles [89]. Finger nails and clothes are also discussed in DeRose et al. [21]. Baraff and Witkin [7] study the numerical stability problem for a mass-spring model of clothes.

Research in this area also overlaps with medical applications $[53,12,67]$. Interactive systems that use soft tissue models and collision detection are required by both applications. Most physically based modeling mentioned above requires so much computation that it is suitable only for non realtime applications. The three 
major problems persist; solving large systems of differential equations quickly, maintaining stability in the solving of these equations and detecting collisions.

\section{Conclusion}

Character animation is still a young subject and much work remains in developing new technologies, as well as in understanding old ones better. The drives in computer animation research would seem to be towards greater interactivity, realism and more robust representations (such as fully adaptive polygonal representations). It seems likely that with greater computing power and new rendering hardware the goals of all these fields will be met.

\section{Acknowledgements}

We thank Robert Kruczera (www.3dcharacters.de) and Taron (www.taron.de) for their character art in Figures 2 and 3 and Jonathon Starck for Figure 5. We also thank the support of the EPSRC (grant number GR/L89518). Finally we would like to acknowledge the help of the Siggraph's graphbib [35].

\section{References}

[1] 3dark. www.3dark.com.

[2] 3dScanners. www.3dscanners.com.

[3] 3dtotal. www.3dtotal.com.

[4] K.B. Atkinson. Close Range Photogrammetry and Machine Vision. Whittles Publishing, 1996.

[5] Christian Babski and Daniel Thalmann. A seamless shape for hanim compliant bodies. VRML 99: Fourth Symposium on the Virtual Reality Modeling Language, pages 21-28, February 1999. ISBN 1-58113-079-1. Held in Paderborn, Germany.

[6] Christian Babski and Daniel Thalmann. 3d graphics define virtual humans on the web. Software Focus, 1(1), 2000.

[7] David Baraff and Andrew Witkin. Large steps in cloth simulation. Proceedings of SIGGRAPH 98, pages 43-54, July 1998. ISBN 0-89791-999-8. Held in Orlando, Florida. 
[8] A. H. Barr. Global and local deformations of solid primitives. Proceedings of SIGGRAPH '84, 1984.

[9] D. Bechmann, Y. Bertrand, and S. Thery. Continuous free form deformations. Proceedings Compugraphics '96, 1996.

[10] P. Besl. Triangles as a primary representation. In Object Representation in Computer Vision (eds. Hebert,M., Ponce,J., Boult,T. and Gross,A.), Lecture Notes in Computer Science 994, Springer, pages 191-206, 1994.

[11] E. Bittar, N. Tsingos, and M-P. Gascuel. Automatic reconstruction of unstructured 3d data: Combining a medial axis and implicit surface. Computer Graphics Forum, 14(3):458—468, 1995.

[12] Morten Bro-Nielsen and Stephane Cotin. Real-time volumetric deformable models for surgery simulation using finite elements and condensation. Computer Graphics Forum, 15(3):57-66, August 1996. ISSN 1067-7055.

[13] E. Catmull and J. Clark. Recursively generated b-spline surfaces on arbitrary topological meshes. Computer-Aided Design, 10:350—355, September 1978.

[14] John E. Chadwick, David R. Haumann, and Richard E. Parent. Layered construction for deformable animated characters. Computer Graphics (Proceedings of SIGGRAPH 89), 23(3):243-252, July 1989. Held in Boston, Massachusetts.

[15] Yu-Kuang Chang and Alyn P. Rockwood. A generalized de Casteljau approach to $3 \mathrm{~d}$ free-form deformation. Proceedings of SIGGRAPH 94, pages 257-260, July 1994. ISBN 0-89791-667-0. Held in Orlando, Florida.

[16] Sabine Coquillart. Extended free-form deformation: A sculpturing tool for 3d geometric modeling. Computer Graphics (Proceedings of SIGGRAPH 90), 24(4):187-196, August 1990. ISBN 0-201-50933-4. Held in Dallas, Texas.

[17] Sabine Coquillart and Pierre Jancéne. Animated free-form deformation: An interactive animation technique. Computer Graphics (Proceedings of SIGGRAPH 91), 25(4):23-26, July 1991. ISBN 0-201-56291-X. Held in Las Vegas, Nevada.

[18] B. Curless and M. Levoy. A Volumetric Method for Building Complex Models from Range Images. In ACM Computer Graphics Proceedings, SIGGRAPH, NewOrleans, USA, pages 303-312, 1996. 
[19] Agnes Daldegan, Nadia Magnenat Thalmann, Tsuneya Kurihara, and Daniel Thalmann. An integrated system for modeling, animating and rendering hair. Computer Graphics Forum (Eurographics '93), 12(3):211-221, 1993. Held in Oxford, UK.

[20] P.E. Debevec, C.J. Taylor, and J. Malik. Modeling and rendering architecture from photographs. In Proc. ACM SIGGRAPH, 1996.

[21] Tony DeRose, Michael Kass, and Tien Truong. Subdivision surfaces in character animation. Proceedings of SIGGRAPH 98, pages 85-94, July 1998. ISBN 0-89791-999-8. Held in Orlando, Florida.

[22] Mathieu Desbrun and Marie-Paule Gascuel. Animating soft substances with implicit surfaces. Proceedings of SIGGRAPH 95, pages 287-290, August 1995. ISBN 0-201-84776-0. Held in Los Angeles, California.

[23] D. Doo and M. Sabin. Behaviour of recursive division surfaces near extraordinary points. Computer-Aided Design, 10:356-360, September 1978.

[24] Nira Dyn, David Levin, and John A. Gregory. A butterfly subdivision scheme for surface interpolation with tension control. ACM Transactions on Graphics, 9(2):160-169, April 1990. ISSN 0730-0301.

[25] H. Edelsbrunner and E. Mucke. Weighted alpha shapes. ACM Transactions on graphics, 13(1):43-72, 1994.

[26] M. Escher, I. Pandzic, and N. Magnenat-Thalmann. Facial deformations for mpeg-4. Computer Animation '98, June 1998. Held in Philadelphia, Pennsylvania, USA.

[27] Petros Faloutsos, Michiel van de Panne, and Demetri Terzopoulos. Dynamic free-form deformations for animation synthesis. IEEE Transactions on Visualization and Computer Graphics, 3(3):201-214, July - September 1997. ISSN 1077-2626.

[28] Farin,G. Curves and Surfaces for Computer Aided Geometric Design: A Practical Guide (2nd edition). Academic Press, 1994.

[29] O. Faugeras. Three-Dimensional Computer Vision - A Geometric Viewpoint. MIT Press, 1993.

[30] A. W. Fitzgibbon, G. Cross, and A. Zisserman. Automatic 3D model construction for turn-table sequences. In R. Koch and L. Van Gool, editors, 3D Structure from Multiple Images of Large-Scale Environments, LNCS 1506, pages 155-170. Springer-Verlag, Jun 1998. 
[31] D. R. Forsey. A surface model for skeleton-based character animation. Eurographics, 1991.

[32] P. Fua and C. Miccio. Animated Heads from Ordinary Images: A Least Squares Approach. Computer Vision and Image Understanding, (to appear), 2000 .

[33] Marie-Paule Gascuel, Anne Verroust, and Claude Puech. Animation and collisions between complex deformable bodies. Graphics Interface '91, pages 263-270, June 1991.

[34] Jean-Paul Gourret, Nadia Magnenat Thalmann, and Daniel Thalmann. Simulation of object and human skin deformations in a grasping task. Computer Graphics (Proceedings of SIGGRAPH 89), 23(3):21-30, July 1989. Held in Boston, Massachusetts.

[35] Graphbib. http://www.siggraph.org/publications/bibliography/.

[36] Sunil Hadap, Endre Bangarter, Pascal Volino, and Nadia MagnenatThalmann. Animating wrinkles on clothes. IEEE Visualization '99, pages 175-182, October 1999. ISBN 0-7803-5897-X. Held in San Francisco, California.

[37] Sunil Hadap and Nadia Magnenat-Thalmann. Interactive hair styler based on fluid flow. Computer Animation and Simulation 2000, pages 87-99, August 2000. ISBN 3-211-83392-7.

[38] Michael Hagenlocker and Kikuo Fujimura. Cffd: a tool for designing flexible shapes. The Visual Computer, 14(5-6):271-287, November 1998. ISSN 0178-2789.

[39] R. Hartley and A. Zisserman. Multiple View Geometry in Computer Vision. Cambridge University Press, 2000.

[40] A. Hilton, D. Beresford, T. Gentils, R. Smith, W. Sun, and J. Illingworth. Whole-body modelling of people from multi-view images to populate virtual worlds. Visual Computer: International Journal of Computer Graphics, 16(7):411—436, 2000.

[41] A. Hilton and J. Illingworth. Geometric fusion for a hand-held 3d sensor. Machine Vision Applications, 12(1):44-51, 2000.

[42] A. Hilton, R. Smith, and W. Sun. Layered animation using displacement maps. In IEEE International Conference on Computer Animation, pages 146-154, May 2000. 
[43] A. Hilton, A.J. Stoddart, J. Illingworth, and T. Windeatt. Reliable surface reconstruction from multiple range images. In 4th European Conference on Computer Vision, pages 117-126. Springer, 1996.

[44] A. Hilton, A.J. Stoddart, J. Illingworth, and T. Windeatt. Implicit surface based geometric fusion. International Journal of Computer Vision and Image Understanding, Special Issue on CAD Based Vision, 69(3):273-291, March 1998.

[45] G. Hirota, R. Maheshwari, and M. C. Lin. Fast volume-preserving free-form deformation using multi-level optimization. Computer-Aided Design, 32(89):499-512, August 2000. ISSN 0010-4485.

[46] H. Hoppe, T. DeRose, T. Duchamp, J. McDonald, and W. Stuetzle. Surface reconstruction from unorganised points. Computer Graphics, 26(2):71-77, 1992.

[47] Hugues Hoppe. Progressive meshes. Proceedings of SIGGRAPH 96, pages 99-108, August 1996. ISBN 0-201-94800-1. Held in New Orleans, Louisiana.

[48] Hugues Hoppe, Tony DeRose, Tom Duchamp, Mark Halstead, Hubert Jin, John McDonald, Jean Schweitzer, and Werner Stuetzle. Piecewise smooth surface reconstruction. Proceedings of SIGGRAPH 94, pages 295-302, July 1994. ISBN 0-89791-667-0. Held in Orlando, Florida.

[49] Doug L. James and Dinesh K. Pai. Artdefo - accurate real time deformable objects. Proceedings of SIGGRAPH 99, pages 65-72, August 1999. ISBN 0-20148-560-5. Held in Los Angeles, California.

[50] T. Kanade and P. Rander. Virtualized reality: Constructing virtual worlds from real scenes. IEEE MultiMedia, 4(2):34-47, 1997.

[51] James T. Klosowski, Martin Held, Joseph S. B. Mitchell, Henry Sowizral, and Karel Zikan. Efficient collision detection using bounding volume hierarchies of k-dops. IEEE Transactions on Visualization and Computer Graphics, 4(1):21-36, January-March 1998. ISSN 1077-2626.

[52] Leif Kobbelt. $\sqrt{3}$ subdivision. Proceedings of SIGGRAPH 2000, pages 103112, July 2000. ISBN 1-58113-208-5.

[53] R. M. Koch, M. H. Gross, F. R. Carls, D. F. von Büren, G. Fankhauser, and Y. Parish. Simulating facial surgery using finite element methods. Proceed- 
ings of SIGGRAPH 96, pages 421-428, August 1996. ISBN 0-201-94800-1. Held in New Orleans, Louisiana.

[54] Rolf M. Koch, Markus H. Gross, and Albert Bosshard. Emotion editing using finite elements. Computer Graphics Forum, 17(3):295-302, 1998. ISSN 1067-7055.

[55] K. Komatsu. Human skin model capable of natural shape variation. NHK Lab. Note (Japan), 329:1, 3-12, March 1986.

[56] Venkat Krishnamurthy and Marc Levoy. Fitting smooth surfaces to dense polygon meshes. Proceedings of SIGGRAPH 96, pages 313-324, August 1996. ISBN 0-201-94800-1. Held in New Orleans, Louisiana.

[57] Aaron Lee, Henry Moreton, and Hugues Hoppe. Displaced subdivision surfaces. Proceedings of SIGGRAPH 2000, pages 85-94, July 2000. ISBN 1-58113-208-5.

[58] Yuencheng Lee, Demetri Terzopoulos, and Keith Waters. Realistic modeling for facial animation. Proceedings of SIGGRAPH 95, pages 55-62, August 1995. ISBN 0-201-84776-0. Held in Los Angeles, California.

[59] J. P. Lewis, Matt Cordner, and Nickson Fong. Pose space deformations: A unified approach to shape interpolation a nd skeleton-driven deformation. Proceedings of SIGGRAPH 2000, pages 165-172, July 2000. ISBN 1-58113208-5.

[60] C. Loop. Smooth subdivision surfaces based on triangles. Master's thesis, Utah University, 1987.

[61] Ron MacCracken and Kenneth I. Joy. Free-form deformations with lattices of arbitrary topology. Proceedings of SIGGRAPH 96, pages 181-188, August 1996. ISBN 0-201-94800-1. Held in New Orleans, Louisiana.

[62] G. Maestri. Digital Character Animation. New Riders Publishing, 1996.

[63] Nadia Magnenat-Thalmann and Daniel Thalmann. Human body deformations using joint-dependent local operators and finite-element theory. Making them move: mechanics, control, and animation of articulated figures, pages 243-262, 1991.

[64] Walter Maurel and Daniel Thalmann. Human shoulder modeling including scapulo-thoracic constraint and joint sinus cones. Computers \& Graphics, 24(2):203-218, April 2000. ISSN 0097-8493. 
[65] R. Mencl. A graph-based approach to surface reconstruction. In EUROGRAPHICS 14(3), pages 446-456, 1995.

[66] R. Mencl and H. Muller. Graph-based surface reconstruction using structures in scattered point sets. In Computer Graphics International, pages 298-311, 1998.

[67] P. Meseure and C. Chaillou. A deformable body model for surgical simulation. The Journal of Visualization and Computer Animation, 11(4):197-208, September 2000. ISSN 1049-8907.

[68] L. Moccozet and N. M. Thalmann. Dirichlet free-form deformations and their application to hand simulation. Computer Animation '97, June 1997. Held in Geneva, Switzerland.

[69] Luciana Porcher Nedel and Daniel Thalmann. Anatomic modeling of deformable human bodies. The Visual Computer, 16(6):306-321, 2000. ISSN 0178-2789.

[70] W. Niem and J. Wingebermuhle. Automatic reconstruction of $3 \mathrm{~d}$ objects using a mobile monoscopic camera. In Proc. IEEE International Conferences on Recent Advances in 3D Digital Imaging and Modeling, Ottawa, Canada, pages 173-180, 1997.

[71] J. Ostermann. Animation of synthetic faces in mpeg-4. Computer Animation '98, June 1998. Held in Philadelphia, Pennsylvania, USA.

[72] Romain Raffin, Marc Neveu, and Frédéric Jaar. Curvilinear displacement of free-form-based deformation. The Visual Computer, 16(1):38-46, 2000. ISSN 0178-2789.

[73] Ravi Ramamoorthi and James Arvo. Creating generative models from range images. Proceedings of SIGGRAPH 99, pages 195-204, August 1999. ISBN 0-20148-560-5. Held in Los Angeles, California.

[74] Ari Rappaport, Alla Sheffer, and Michel Bercovier. Volume-preserving freeform solids. IEEE Transactions on Visualization and Computer Graphics, 2(1):19-27, March 1996. ISSN 1077-2626.

[75] B. Roehl. Draft Specification for a Standard VRML Humanoid, Version 1.0. http://ece.uwaterloo.ca/ h-anim/, 1997.

[76] M. Rutishauser, M. Stricker, and M. Trobina. Merging range images of arbitrarily shaped objects. In Proceedings of IEEE Conference on Computer Vision and Pattern Recognition, pages 573-580, 1994. 
[77] Thomas W. Sederberg and Scott R. Parry. Free-form deformation of solid geometric models. Computer Graphics (Proceedings of SIGGRAPH 86), 20(4):151-160, August 1986. Held in Dallas, Texas.

[78] Jianhua Shen and Daniel Thalmann. Interactive shape design using metaballs and splines. Implicit Surfaces '95, April 1995.

[79] M. Soucy and D. Laurendeau. A general surface approach to the integration of a set of range views. IEEE Trans. Pattern Analysis and Machine Intelligence, 14(4):344-358, 1995.

[80] W. Sun, A. Hilton, R. Smith, and J. Illingworth. Building layered animation models from captured data. In Eurographics Workshop on Computer Animation, pages 145-154, September 1999.

[81] W. Sun, A. Hilton, R. Smith, and J.Illingworth. Layered animation of captured data. Accepted for publication in Visual Computer: International Journal of Computer Graphics, 17(4), 2001.

[82] Demetri Terzopoulos and Hong Qin. Dynamic nurbs with geometric constraints for interactive sculpting. ACM Transactions on Graphics, 13(2):103136, April 1994. ISSN 0730-0301.

[83] N. M. Thalmann, S. Carion, M. Courchesne, P. Volino, and Y. Wu. Virtual clothes, hair and skin for beautiful top models. Computer Graphics International 1996, 1996.

[84] G. Turk and M. Levoy. Zippered Polygon Meshes from Range Images. In ACM Computer Graphics Proceedings, SIGGRAPH, Orlando, Florida, pages 311-318, 1994.

[85] Russell Turner and Enrico Gobbetti. Interactive construction and animation of layered elastically deformable characters. Computer Graphics Forum, 17(2):135-152, 1998. ISSN 1067-7055.

[86] Pascal Volino and Nadia Magnenat-Thalmann. Real-time simulation of clothes. Computer Graphics International 2000, June 2000. Held in Geneva, Switzerland.

[87] A. Watt and M. Watt. Advanced Animation and Rendering Techniques. ACM press, 1999.

[88] Jane Wilhelms and Allen Van Gelder. Anatomically based modeling. Proceedings of SIGGRAPH 97, pages 173-180, August 1997. ISBN 0-89791896-7. Held in Los Angeles, California. 
[89] Yin Wu, Prem Kalra, Laurent Moccozet, and Nadia Magnenat-Thalmann. Simulating wrinkles and skin aging. The Visual Computer, 15(4):183-198, 1999. ISSN 0178-2789.

[90] Denis Zorin, Peter Schröder, and Wim Sweldens. Interpolating subdivision for meshes with arbitrary topology. Proceedings of SIGGRAPH 96, pages 189-192, August 1996. ISBN 0-201-94800-1. Held in New Orleans, Louisiana. 